\title{
Fascia Adherens
}

National Cancer Institute

\section{Source}

National Cancer Institute. Fascia Adherens. NCI Thesaurus. Code C13985.

Fascia adherens, similar to a zonula adherens, is a half $\mathrm{Z}$ line found in the cardiac muscle as one of the three intercalated disks. They anchor actin filaments from terminal sarcomeres and transmit contractile force between myocytes. ( $\mathrm{NCl})$ 